\title{
Microanalysis of the Cross-sectional Surface of 3D Printed NiMnGa Magnetic Shape Memory Alloy Using Electron Microscopy and Nanoindentation
}

\author{
Constantin Solomon ${ }^{1}$, Yash Trivedi ${ }^{1}$, Christopher Bansah ${ }^{1}$ and Matthew Caputo ${ }^{2}$ \\ ${ }^{1}$ Youngstown State University, Youngstown, Ohio, United States, ${ }^{2}$ PennState Shenango, Sharon, \\ Pennsylvania, United States
}

Polycrystalline NiMnGa parts with complex geometries produced 3D printing show reversible magneticfield-induced strain of $0.01 \%$ [1,2]. The reversible magnetic-field-induced strain can be improved by controlling morphological defects induced during sample synthesis, sample's chemical composition, and mechanical properties. In order to correlate the synthesis conditions (3D printing and sintering parameters) and the part behavior under the magnetic field with the microstructural characteristics, microanalysis of the cross-sectional surfaces was performed using SEM, EDS, FIB, and nanoindentation techniques. Nanoindentation is a suitable method to map-out mechanical behavior of materials within small volumes [3]. In this work, nanoindentaion was employed to investigate local mechanical properties of interest such as hardness, modulus of elasticity, yield strength, and fracture toughness. Previous nanoindenation studies on shape memory alloys reported on mechanical anisotropy, time dependent mechanical properties, and local shape memory properties as function of temperature [46].

The 3D printed NiMnGa parts (Ni50.9Mn30.7Ga18.4 at\%) were prepared from ball milled powders using binder-jet technology. After printing the parts were sintered in argon atmosphere at $1080^{0} \mathrm{C}$ for different time periods ( $24 \mathrm{~h}, 40 \mathrm{~h}$, and $50 \mathrm{~h}$ ). Nanoindenation was performed on electrochemical etch cross-sections using both Berkovich and flat tip indentors. Hardness and modulus of elasticity were calculated from loading-unloading curves obtained during nanoindentation using Berkovich indentor (maximum load of $250 \mathrm{mN}$ ). Yield strength was calculated, with the aid of a Matlab code, from loading-unloading curves produced by a flat tip indentor (maximum load ranging from 50 to $400 \mathrm{mN}$ ). Fracture toughness was calculated using Laugier's model [7].

SEM investigation of the cross-sectioned surface of 3D printed samples shows the existence of pores in all samples, irrespective of the sintering time, Fig. 1 (a) to (c). The pores presence is expected in binderjet 3D printed samples and enhances the reversible magnetic-field-induced strain, in the case of NiMnGa alloys [1]. From SEM micrographs is clearly seen that the porosity decreases with increasing sintering time. Chemical analysis using EDS indicate uniform chemical composition.

Sintering time have no effect on hardness and electric modulus of printed samples. The average hardness was determined to be $1.97 \mathrm{GPa}$, while the calculated average modulus of elasticity was 33.67 GPa. Yield strength was determined using nanoindenation with a flat circular tip indentor $(20 \mu \mathrm{m}$ diameter $)$. Due to the porosity, this nanoindentation was more difficult to perform as compared to the sharp indenter. The loading and unloading curves does not overlap each other, which confirms the material's plastic deformation during indentation, Fig. 2. The loading-unloading plot geometry depends on both the maximum load applied and individual grain orientation. The average yield strength was determined to be 545.75 MPa.

Fracture toughness is defined as material resistance to rapid crack propagation. In order to understand material's micro-fracture during indentation with a sharp indenter one model uses an approximation for tensile stress distribution in the elastic/plastic indentation field [7]. The model takes into account the relationship between the size of a critical flaw and the load required for crack propagation. In the case of 3D printed parts, due to the brittle nature of NiMnGa materials, micro-fracture following Palmqvist crack 
propagation was considered to occur during nanoindentation with sharp Berkovich indenter, Fig. 1 (d). The maximum force of $400 \mathrm{mN}$ was applied. For the assessment of crack type propagation below the surface the FIB was used. For the $24 \mathrm{~h}$ sintered samples the fracture toughness was determined to be 1.841 $\mathrm{Pa}(\mathrm{m})^{1 / 2}$. It was interesting to observe that the fracture toughness of printed samples decreased with increasing the sintering time. The reason for this behavior is still under investigation. One possible explanations might be related to hydrogen embrittlement.
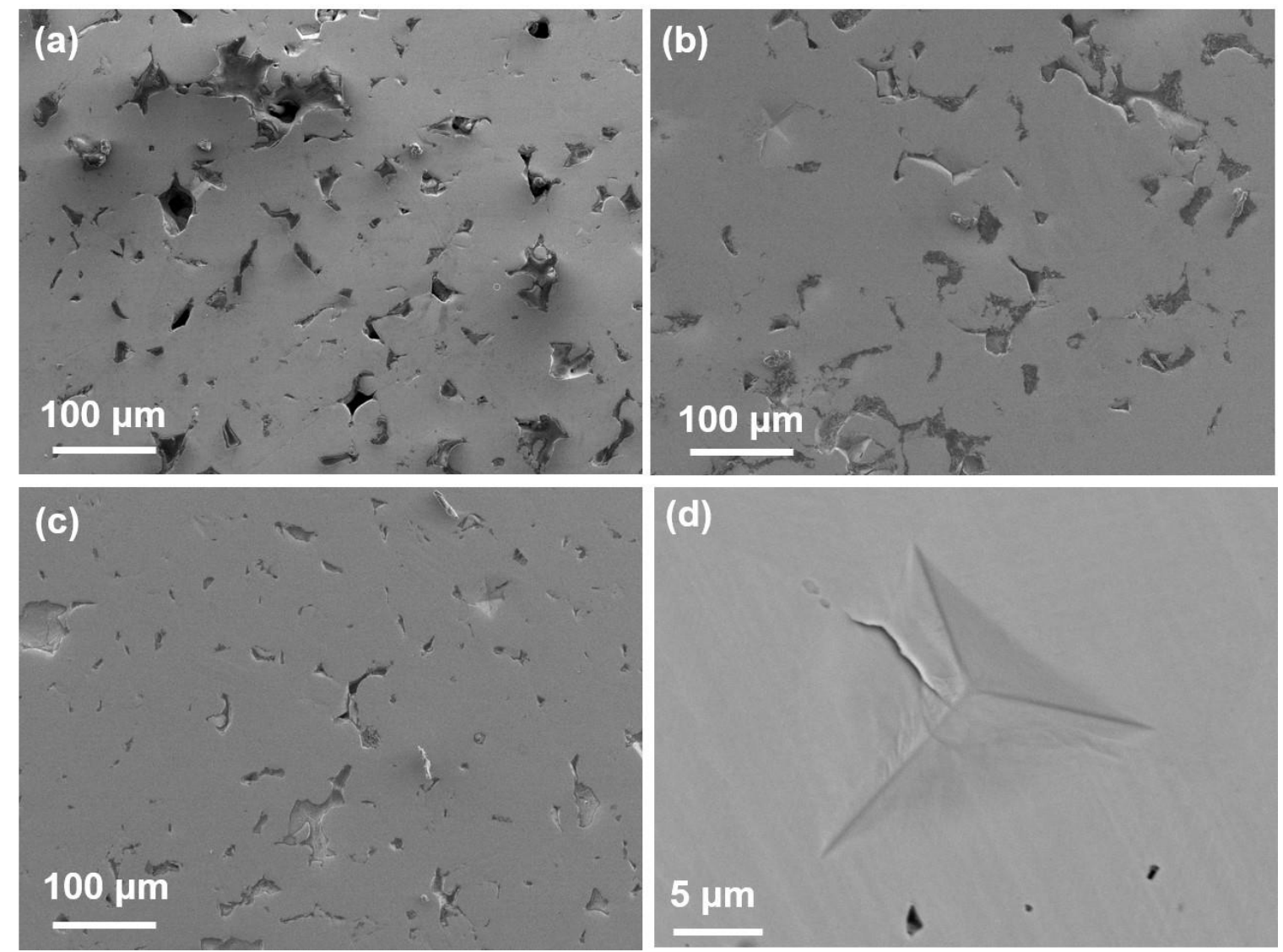

Figure 1. (a) - (c) Secondary electron micrographs of the cross-sectional surfaces of binder-jet 3D printed NiMnGa parts sintered at $10800 \mathrm{C}$ for $24 \mathrm{~h}, 40 \mathrm{~h}$, and $50 \mathrm{~h}$, respectively. The porosity decreases with increasing sintering time. (d) SEM micrograph of a Berkovich indent profile on the surface of the sample sintered for $50 \mathrm{~h}$. The measured crack length was used for calculating fracture toughness using Laugier's model. 


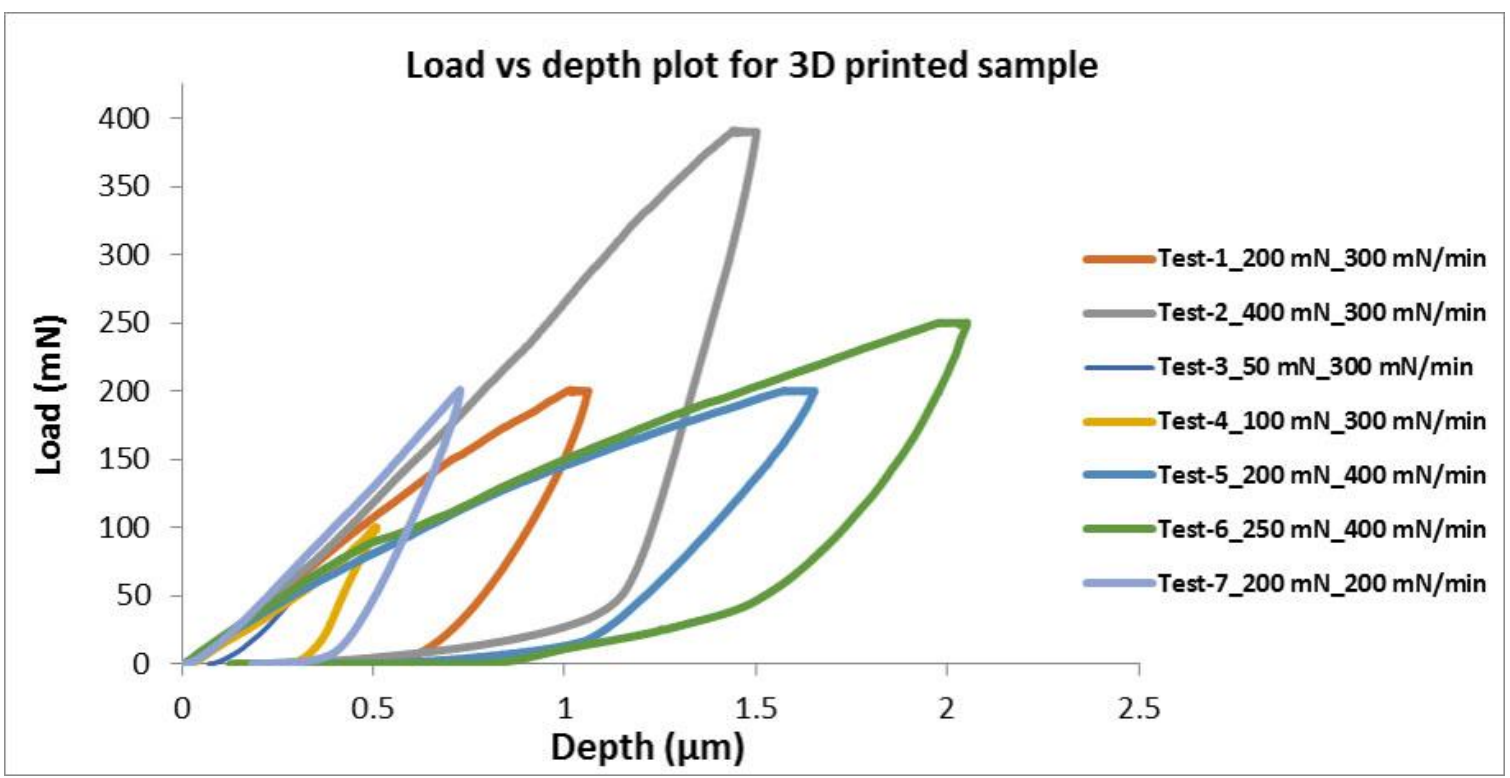

Figure 2. Flat tip indentation responses of typical load-displacement relationships under selected loading conditions for a 3D printed sample sintered at 10800C for $24 \mathrm{~h}$.

\section{References}

[1] M.P. Caputo, A.E. Berkowitz, A. Armstrong, P. Muller, and C.V. Solomon, 4D printing of net shape parts made from Ni-Mn-Ga magnetic shape memory alloys, Additive Manufacturing 21 (2018) 579 - 588. [2] M.P. Caputo and C.V. Solomon, A facile method for producing porous parts with complex geometries from ferromagnetic Ni-Mn-Ga shape memory alloys, Materials Letters 200 (2017) 87 - 89.

[3] W.C. Olivier and G.M. Pharr, An improved technique for determining hardness and elastic modulus using load and displacement sensing indentation experiments, Journal of Materials Research 7 (1992) $1564-1583$.

[4] A. Jayaraman, M.S.R.N. Kiran, and U. Ramamurty, Mechanical anisotropy in austenitic NiMnGa alloy: nanoindenation studies, Crystals 7 (2017) 254.

[5] D.K. Satapathy and S. Aich, Time dependent nanomechanical properties of NiMnGa Heusler alloy, Journal of Alloys and Compounds, 788 (2019) 10 - 20.

[6] P. Li, H.E. Karaca, and Y.T. Cheng, Rapid characterization of local shape memory properties through indentation, Scientific Reports 7 (2017) 14827.

[7] B.R. Lawn and A.G. Evans, A model for crack initiation in elastic/plastic indentation fields, Journal of Materials Science 12 (1977) 2195 - 2199. 